\title{
Virtual Sport-Based Positive Youth Development During the COVID-19 Pandemic
}

\author{
Samantha Bates ${ }^{1}\left[0\right.$. Dekia Greene ${ }^{2} \cdot$ Luke O'Quinn $^{3}$
}

Accepted: 5 May 2021 / Published online: 15 May 2021

(c) The Author(s), under exclusive licence to Springer Science+Business Media, LLC, part of Springer Nature 2021

\begin{abstract}
Sport is a foundational context for social, emotional, physical, and psychological development. The COVID-19 pandemic displaced many youth from their normative sport activities. As a result, sport-based positive-youth development (PYD) programs, typically delivered in person, had to reimagine ways to reach and engage youth in sport and life skill development. In fall 2020, The Los Angeles Football Club Youth Leadership Program (LAFC YLP) developed seven virtual sport-based PYD videos and one workshop for 120 socially vulnerable youth and their families. All virtual activities were designed to teach life skills through sport and play. Our study sought to explore the accessibility of the virtual sport-based PYD activities, the lived experiences of youth participants during lockdown, and learning outcomes of youth and families who participated in the program. We developed a mixed methods study using an online survey and virtual platform to allow youth to share photos, draw pictures, and leave comments about their lived experiences. Our findings indicated 53 youth and their families participated in the virtual sport-based PYD program and reported the activities were accessible, enjoyable, and challenging for the youth participants. In addition, 26 youth shared photos, images, or posts about their lived experiences. Our thematic analysis of the photos, images, and posts indicated the virtual sport-based PYD activities facilitated positive emotional responses, positive peer interaction, engagement with family, and utilization of environmental resources during the COVID-19 pandemic. Importantly, our findings also suggest virtual sport-based PYD activities may facilitate life skill transfer; an important developmental mechanism for learning in lieu of the decreased opportunities for sport and social interaction during the COVID-19 pandemic.
\end{abstract}

Keywords Sport-based positive youth development $\cdot$ Virtual programming $\cdot$ COVID-19 pandemic $\cdot$ Access $\cdot$ Life skill transfer

\section{Introduction}

The global COVID-19 pandemic has influenced children's ability to engage in sport in schools and communities (Bachman, 2020; Dunton et al., 2020; Sanderson \& Brown, 2020; The Aspen Institute, 2020). Barriers such as transportation, space, time, costs, availability, social distancing policies, and family hardships have changed the landscape

Samantha Bates

bates.485@osu.edu

1 College of Social Work, The Ohio State University, 1947 College Rd N, Columbus, OH 43210, USA

2 Department of Social Work, Texas Christian University, Fort Worth, TX, USA

3 Bresee Youth Center, Los Angeles, CA, USA for participation and engagement in sports. In fact, a recent report from The Aspen Institute (2020) indicated families and children are allocating less time per week now than before the pandemic toward active engagement in sports. Additionally, only three out of ten youth who played sports prior to the pandemic expressed an interest in participating in sports (The Aspen Institute, 2020). These trends have likely resulted from online schooling and the cancellation of many community activities, practices, and camps, leaving youth and families with a sense of loss and fewer opportunities to engage in physical activity and sport.

The COVID-19 pandemic also impacted disparities in access to sport. Prior to COVID-19, youth with limited socioeconomic means and youth of color means were less likely to be able to afford sport or access recreational facilities in their schools and communities (The Aspen Institute, 2019; Whitaker et al., 2019). Recent reports suggest disparities are 
increasing due to the COVID-19 pandemic. For instance, The Aspen Institute (2020) found youth from socially vulnerable backgrounds are less likely to receive virtual training activities from their community sports programs compared to their more affluent peers. Due to multi-faceted, intersecting, and overlapping risk factors, complicated further by the COVID-19 pandemic, some of our nation's most vulnerable youth face increased risks for sedentary behaviors and heightened behavioral health concerns (Dunton et al., 2020; Margaritis et al., 2020; Rundle et al., 2020).

Traditionally, summer camps, afterschool programs, and community-based recreation settings house sport-based positive youth development (PYD) programs that seek to leverage sport and play to teach life skills, and all of these program activities tend to take place in person. One gap in the current body of research on sport-based PYD is whether virtual sport activities can reach and engage youth in life skill development during the COVID-19 pandemic. Our research study sought to explore whether virtual sport-based PYD activities were accessible to a population of socially vulnerable Latinx youth and their families during the COVID-19 pandemic. We also sought to examine the lived experiences and learning outcomes of youth and families participating in the virtual sport-based PYD activities. Our findings aim to contribute to what researchers know about strategies and interventions that not only reach, but also leverage sport to promote positive developmental outcomes during the COVID-19 pandemic.

\section{Youth Sport and COVID-19}

Researchers contend participation in sport supports positive developmental outcomes for children and adolescents (Whitley et al., 2019). Sport programs that encourage physical activity and knowledge of healthy behaviors can promote children's overall health and well-being into adulthood (Bouchard et al., 2012). Researchers also argue that participation in sport has psychological benefits above and beyond those seen with physical activity because of its social nature. For instance, engagement in sports and play activities facilitates improved self-esteem and increased social skill development (Durlak et al., 2010; Hermens et al., 2017). When intentionally designed and accessible, sport settings provide youth with meaningful access to physical activity and opportunities to build social and personal skills (Holt et al., 2017; Whitley et al., 2019).

The COVID-19 pandemic has made it more challenging for youth to access the positive developmental assets embedded in sport. At the community level, youth sports buffer community risks and provide settings that help to build protective factors. McMahon et al. (2013) claimed that living in communities with high rates of poverty, crime, and chronic neighborhood problems exposes children to extraordinary stressors and generally linked with negative outcomes. The aforementioned outcomes include drug and alcohol use, delinquency, criminal activities, and early sexual activity (Center for Disease Control, 2021). Scholars found that children's involvement in sports programming can buffer risks for substance misuse and such complex social issues in communities as early sexual activity, delinquency, and crime (Anderson-Butcher, 2019; Spruit et al., 2016). Due to the cancellation of community sport programs, the protective community elements embedded in sport may be less accessible during the COVID-19 pandemic, especially for youth from socially vulnerable backgrounds.

At the family level, the pandemic has exacerbated inequities in access to sport on the basis of socioeconomic status (SES). For example, families with greater job security and higher SES are more likely to live and work in communities with the resources to engage their children in physically distanced play and safe sport activities (i.e., playgrounds, parks, school facilities, equipment, etc.; Kelly et al., 2020). Meanwhile, youth and families from lower SES backgrounds have less access to infrastructure in their schools and communities that allow for safe involvement in sport. In turn, youth and families from lower SES backgrounds may not only be those with less access due to broader structural factors, but also more likely to utilize non-traditional spaces and activities to engage their children in sport and play during the lockdown (Kelly et al., 2020).

At the individual level, sports also are a hook for involvement in physical activity and social connection, especially when relevant to the racial, ethnic, or cultural identity of children and their families. Regular physical activity is highly beneficial for youth to build and maintain healthy muscles and bones, promote psychological well-being, and support academic success (Hermens et al., 2017; Janssen $\&$ LeBlanc, 2010). Due to COVID-19 restrictions, including the closures of schools, parks, recreation centers, and the cancellation of youth sports, youth are at an increased risk for sedentary behaviors that influence their health, well-being, and academic performance (Dunton et al., 2020). Moreover, scholars argue sport contexts are central to social skill development and help youth build relationships with peers, other families, sport coaches, and other caring adults (Holt et al., 2017; Jones et al., 2017). Without in-person youth sport and play activities, youth have fewer opportunities to build relationships and access additional social support.

\section{Sport-Based Positive Youth Development}

Positive youth development (PYD) is grounded in the belief that all youth have the potential for successful, healthy development. PYD programs are often grounded in the "Big 3" 
characteristics: (a) positive and sustained adult-youth relationships, (b) life skill building activities, and (c) opportunities for youth to participate in community activities (Lerner, 2004). Life skill building is an essential feature of PYD programs that can foster positive developmental outcomes for youth (Lerner, 2004). The development of life skills during childhood and adolescence helps to promote positive longterm development and buffer psychosocial risks (Botvin \& Griffin, 2004; Pierce et al., 2017). Unlike traditional PYD programming, sport-based $\mathrm{PYD}$ programs leverage the "Big 3 " to teach life skills using sport as the hook for engagement and participation.

Through the use of intentionally designed sport programming, sport-based PYD programs serve a dual purpose: addressing the development of positive psychological and social skill outcomes for youth (e.g., life skills, prosocial behaviors, and social competence) and promoting positive health-related outcomes (e.g., fitness, optimal body composition, interest in physical activity; Anderson-Butcher et al., 2013; Whitley et al., 2019). Life skills are intrapersonal and interpersonal assets that enable individuals to manage the stressors, challenges, and demands of everyday life (Camiré et al., 2012). Sport-based PYD programs teach life skills by modeling skills in sport, positively reinforcing the skills, providing youth with opportunities to practice the skills, and debriefing the activities with group discussions of how youth can use the skills outside of sport (Bean et al., 2016; Pierce et al., 2018).

In recent studies, scholars imply teaching life skills in sport contexts can foster the development of transferable skills; however, gaps remain in our understanding of how life skills develop in sport. Researchers contend gaps exist in regard to whether implicit or explicit sport-based program design features best facilitate transference of life skills to other settings (Turnnidge et al., 2014). Moreover, scholars argue solely quantitative assessments of sport-based PYD programs make assumptions about what skills youth develop over time. Newman (2020) recently called for sport-based PYD researchers to "construct knowledge from the lived experiences of youth who are currently experiencing these outcomes (Newman, 2020, p. 645)." Newman's (2020) case study and photo-elicitation methodologies move the field toward greater understanding of youths' lived experiences and the utility of sport-based PYD to support life skill transfer (i.e., the transfer of skills learned in sport to other settings). However, Newman (2020) argues studies with larger and diverse samples as well as rigorous design methods can enhance our understanding of life skill development in sport.

\section{Virtual Sport-Based PYD During COVID-19}

The COVID-19 pandemic has altered the ways in which sport and sport-based PYD programs can reach youth, especially if programming is unable to take place in person. Intersectional risk factors may exacerbate risks for isolation and widen disparities in access to sport for youth and their families. For instance, Latinx families in socially vulnerable communities in particular often see soccer as more than a sport and context for physical activity and rather a cultural rite of passage (Nadel, 2014). Latinx communities often leverage soccer as a tool to support spiritual, physical, emotional, and social development (Nadel, 2014). When sports such as soccer are unavailable, Latinx youth and their families may have fewer opportunities to engage in physical activity and to access activities that facilitate a sense of cultural pride and connection therein exacerbating children's risks for poor psychological and social health during lockdown.

As a result of the COVID-19 pandemic, sport-based PYD programs have had to reimagine ways to reach youth in order to maximize their opportunities for positive developmental outcomes. We know schools pivoted to delivering online teaching and learning during the COVID-19 pandemic, yet we know little about whether the teaching and learning that happens in sport-based PYD programs can take place in online spaces. At present, the use of virtual sport and play activities is a relatively new and ever-evolving approach as sport leaders try to retain and reach youth during the COVID-19 pandemic (Kelly et al., 2020). Scholars indicate sport programs are attempting to offer online streaming services whether via live or recorded activity classes for youth using platforms such as Zoom, YouTube, and Instagram (Dunton et al., 2020; Kelly et al., 2020; Sherwin, 2020). To date, however, no studies to our knowledge have explored whether virtual sport-based PYD activities can reach socially vulnerable youth and their families.

The COVID-19 pandemic presents scholars with opportunities to explore the accessibility and learning outcomes of youth who participate in virtual sport-based PYD activities. Social work scholars also recognize and value the lived experiences of youth and families. Studies that elevate the voices and perspectives of youth participating in sport-based PYD can strengthen our understanding of life skill development in sport. To address these areas of research, our study sought (a) to explore the accessibility of sport-based PYD activities to a population of socially vulnerable youth and their families during the COVID-19 pandemic and (b) to analyze the lived experiences and learning outcomes of youth and families who participated in these activities.

\section{Method}

\section{Study Context}

The context of the current study was in Los Angeles (LA), California, at the Bresee Youth Center. The Bresee Youth 
Center is located in one of the most at-risk neighborhoods in LA. With 44,000 people per square mile, this neighborhood has one of the densest populations west of the Mississippi. According to the LA Police Department, over 800 active gang members live in the community surrounding Bresee. The community surrounding the Bresee Youth Center is also the part of LA with the highest concentration of recently arrived immigrants from Mexico and Central America (Bresee Youth Center, 2020). In 2017, The Bresee Youth Center partnered with the Los Angeles Football Club (LAFC) to create, support, and fund The Los Angeles Football Club Youth Leadership Program (LAFC YLP).

Within the Bresee Youth Center's programming, The LAFC YLP functions as a sport-based PYD program designed to prevent gang membership and to improve postsecondary education outcomes for high school youth. In addition, the LAFC YLP seeks to harness the power of soccer to create high school role models and mentors for younger youth in the Los Angeles community. Prior to the COVID-19 pandemic, high school youth in the LAFC YLP taught life skills to younger youth by hosting communitybased soccer classes. This ladder to leadership approach allows high school youth to practice their life skills while also serving as positive role models to younger youth in the community.

A consequence of the COVID-19 pandemic included the inability for high school youth in the LAFC YLP to provide in-person soccer classes to younger youth in the community. Instead, high school youth and program leaders created virtual sport-based PYD videos and an online workshop to reach and engage younger youth in sport activities. Youth and families who received the invitations to participate in the virtual sport-based PYD program (i.e., activities and workshops) were those already in partnership with the Bresee Youth Center. The center also sent youth and families a scorecard to track their engagement in the program and invited youth and their families who participated in the program to pick up prizes from the Bresee Youth Center (see Fig. 1).

In the fall of 2020, high school youth leaders sent seven virtual sport-based PYD activity videos to approximately 120 youth and families in the greater LA community over the course of three months. High school youth also led one live 90-min virtual workshop for 40 youth and their families. All seven of the sport-based PYD activities and the virtual workshop focused on one life skill that aligns with the LAFC YLP program pillars (i.e., commitment, accountability, community, or integrity). In addition, high school leaders modeled and positively reinforced each of the virtual activities to the youth and their families, and all activities included several debrief questions. Our study sought to utilize a mixed methods approach to explore the accessibility and experiences of youth participating in the aforementioned activities.

\section{Quantitative Approach}

\section{Procedure and Sample}

The lead author's Institutional Review Board approved all study procedures. To collect quantitative data, adult program leaders of the LAFC YLP invited youth and families to participate in brief online surveys via email. The authors designed online surveys (a) to assess whether youth and their families participated in the activities and (b) to explore youths' lived experiences and learning outcomes when they participated in the virtual sport-based PYD activities.

\section{Online Survey}

The online survey included ten questions. The first question asked youth and families to share the child's age and how many sport-based PYD activities they participated in ranging from "1" to "4 or more." Notably, we did not capture any additional demographic information based on recommendations from the youth center to protect the youth and families utilizing their services (i.e., socially vulnerable and undocumented youth and families). Bresee staff estimated $90 \%$ of youth and their families invited to participate identified as Latinx and were those living at or below the poverty line. We also asked youth and their families to respond to seven questions about their perceptions of the virtual sport-based PYD activities. We measured questions on a 3-point Likert scale $(1=$ No $; 2=$ Maybe $; 3=$ Yes $)$. Example questions included: "I feel proud to be a part of this program" and "What we do in this program is important to me." We also included one open-ended question on the survey that asked how, if at all, youth could use what they learned in the virtual sport-based PYD activities in other areas of their lives.

\section{Data Analysis}

We used descriptive statistics (i.e., means and frequencies) to examine the average age of youth participants, the average number of sport-based PYD activities youth and families participated in, and responses to items on the survey. We also used Dedoose software and thematic analysis to explore responses to the open-ended question on the survey related to how youth could use what they learned via the activities in other areas of their lives (Lincoln \& Guba, 1986).

\section{Qualitative Approach}

\section{Procedures and Data Collection}

Adult program leaders also sent parents and guardians another email that included an informed consent form for their child to participate in a photo and drawing component 


\section{Fall 2020 YLP Virtual Scorecard}

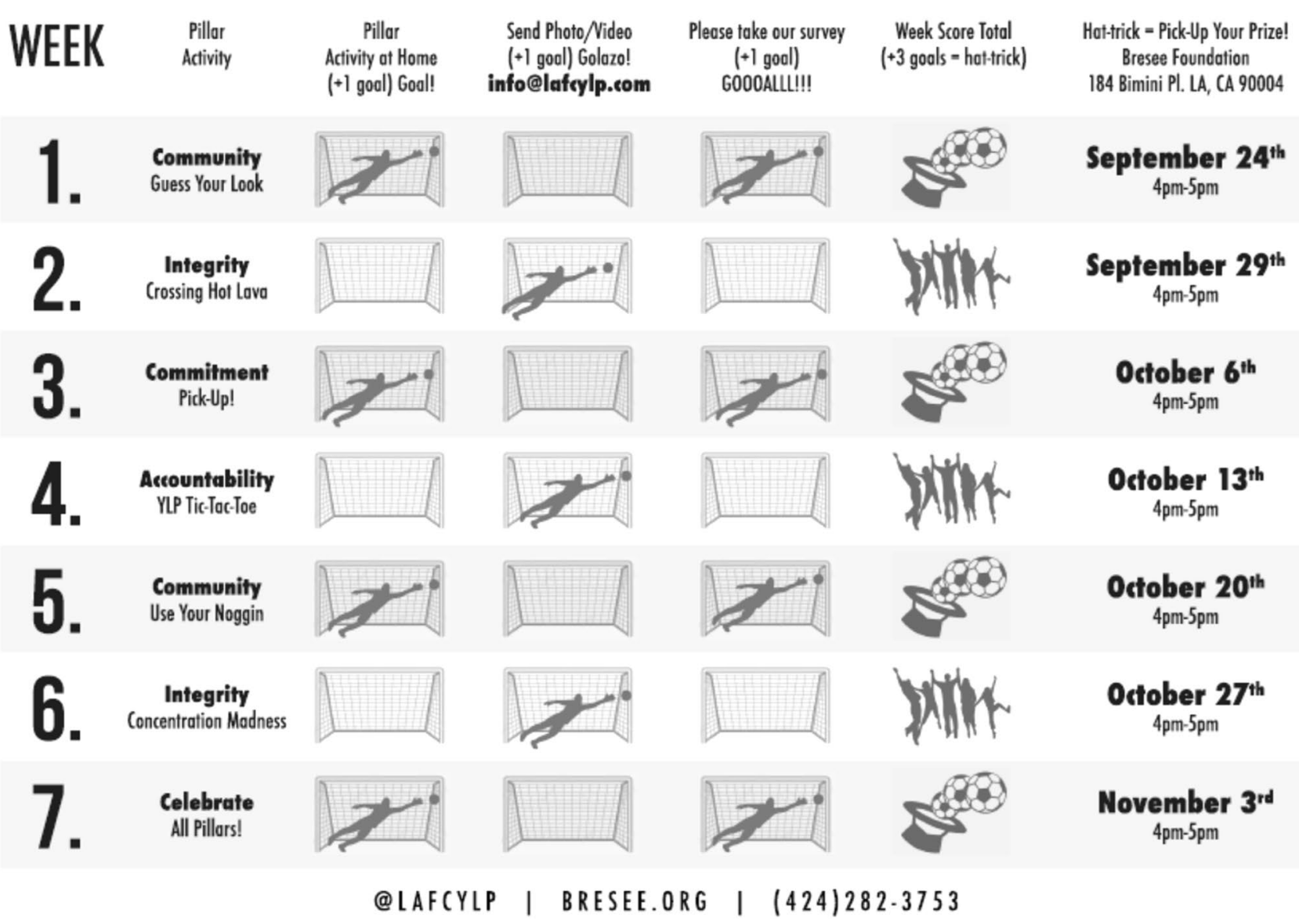

Fig. 1 Fall 2020 virtual scorecard

of the study using the online platform Padlet (padlet.com). Padlet is an online platform where children can draw, upload pictures, and journal their ideas. We recommended children be at least 6 years of age or older and incentivized this component of the study by offering one family the opportunity to win a $\$ 100$ online Amazon gift card. Within the consent form, we included a Padlet link. In the Padlet, we posted an audio file in both English and Spanish that detailed how to use the platform and reiterated elements of our informed consent documents including notions of safety, assent, and use of the photos, comments, and drawings for research purposes. For anonymity purposes, we again did not capture any demographic information to protect the families in association with the youth center.

In the Padlet, we asked youth and their families to respond to five questions: (a) How do the videos make you feel?; (b) LAFC has four pillars! Community, integrity, accountability, and commitment. Take or post a picture of where you might use these pillars; (c) Take or post a picture of where you play when watching the videos; and, (d) What was your favorite video? (write a post, take a picture, or draw). Within the Padlet platform, youth had the option to upload photos (of themselves or chose one off the internet if they did not want to post a personal photo), draw pictures, write in the text boxes, and like one another's posts.

\section{Data Analysis}

Prior to engaging in data analysis, we reflected on our biases and social positionality (i.e., one White female researcher and another Black female research assistant) given reflexivity is an important component of qualitative inquiry. Notably, we recognized we were not members of the communities in which we were studying and discussed approaching individuals as experts of their own experiences. Further, we discussed how the Padlet platform was an interactive tool that allowed children and families to share their lived experiences with little intervention on our behalf. We then 
analyzed photos, comments, and drawings from youth using a thematic analysis (Lincoln \& Guba, 1986). A thematic analysis approach allowed us to examine the experiences and learning outcomes from the lens of the youth via their responses, drawings, comments, or photos.

We started by reviewing each photo, comment, or drawing individually and creating general themes. We then identified broader themes using inductive processes, allowing for youths' posted photos and drawings to frame our understanding of these data. Our process was iterative, allowing us to modify and reshape themes until we reached interrater agreement and thematic consensus. We used triangulation (i.e., cross-checking) and an expert peer to debrief findings to enhance credibility, decrease bias, and ensure validity (Lincoln \& Guba, 1986). The expert peer who supported the debriefing process was a program leader with LAFC YLP who had familiarity with the philosophy and mission of the program and years of experience working with Latinx youth in the LA community.

\section{Quantitative Results}

In total, 53 youth and their families completed the online surveys. Participants' ages ranged from 6 to $13(\mathrm{M}=9$ years old). Our findings indicated the average number of sportbased PYD activity videos watched by youth participants and their families was approximately three videos. Table 1 details the frequencies and means on each question used to assess perceptions of the virtual sport-based PYD activities. Results indicated youth and their families felt proud to be a part of the program, youth felt excited about what they were learning, and youth perceived the activities to help them learn new and challenging skills.

\section{Life Skill Transfer}

The survey asked youth and their families to describe how, if at all, they could use what they learned from the virtual sport-based PYD activity videos or workshop in their lives. Three themes emerged from these responses (see Table 2). The first theme included learning new ways to engage in physical activity while at home. The second theme focused on using the skills learned via the virtual sport-based PYD activity videos in school. For example, youth shared the sport-based PYD activity videos taught them skills that could help them pay attention or follow directions. Lastly, youth shared the activities taught them ways to use sports, specifically soccer, to build relationships with others in their communities.

\section{Qualitative Results}

In total, 26 youth and their families consented to participate in the qualitative part of the study. We identified five themes that described youths' lived experiences and learning outcomes after engaging in the virtual sport-based PYD activities during the COVID-19 pandemic. The five themes included: (a) positive emotional responses; (b) positive peer
Table 1 Perceptions of virtual sport-based PYD activities $(\mathrm{N}=53)$

\begin{tabular}{lllll}
\hline Question & No! (\%) & Maybe (\%) & Yes! (\%) & Mean \\
\hline I feel proud to be a part of this program & 0 & 0 & 100 & 3.00 \\
What we did in the program will help me succeed & 0 & 4 & 96 & 2.96 \\
I feel excited about what I learned today & 0 & 2 & 98 & 2.98 \\
The program helped me try new things & 0 & 2 & 98 & 2.98 \\
The program helped me build new skills & 0 & 6 & 94 & 2.94 \\
What we do in this program is important to me & 0 & 5 & 95 & 2.95 \\
What we do in this program is challenging in a good way & 0 & 2 & 98 & 2.98 \\
\hline
\end{tabular}

Table 2 Life skill transfer themes

\begin{tabular}{ll}
\hline Theme & Quotes \\
\hline Engagement in Physical Activity at Home & "It helped me be active at home" \\
& "You can do a lot of exercise at home without leaving your house" \\
Following Directions or Rules at School & "I will pay attention to my teacher" \\
Building Community Relationships & "I can use this to follow the directions of the teachers" \\
& "I can use it in my neighborhood so everyone can live better" \\
& "I learned that I can play soccer with my community and get to \\
\hline
\end{tabular}


interactions; (c) engagement with family; (d) utilization of environmental resources; and, (e) life skill transfer.

\section{Positive Emotional Responses}

Several youth reported positive emotional responses when asked about their experiences with the virtual sport-based PYD activities (see Fig. 2). Youth described a series of emotions associated with the virtual sport-based PYD videos with the most common responses being positive emotions like "feel good" and "so fun." A research participant also drew an image of a child smiling.

\section{Positive Peer Interactions}

The online data collection platform allowed youth to validate and support their peers by liking one another's photos, drawings, or comments. Although not central to this study, we found youth were highly supportive of their peers in the online Padlet platform. In fact, six images, comments, or drawings received positive endorsements via the "like" button indicating engagement and positive peer interactions online. Notably, common themes across the comments or images "liked" by peers included endorsement of fun activities shared by the sport-based PYD program, outdoor spaces, and statements about engagement in activities with family members (see Fig. 3).

\section{Engagement with Family}

Several of the youth posted about engaging in the sportbased PYD activities with their families. Youth described playing with their "dad" and "family." Youth also shared where they played such as "in my sisters backyard" and "in my back yard." Having someone to play with and a space to play were both central to participation in the virtual sport-based PYD activities (see Fig. 4).

\section{Utilization of Environmental Resources}

Another theme showed that youth utilized various resources in their environments when engaging in the sport-based PYD activities during the COVID-19 pandemic. Several youth shared photos or images of backyards, indoor home spaces, and an outdoor concrete backyard area when reflecting on where they participated in sport and the virtual sportbased PYD activities (see Fig. 5).

\section{Life Skill Transfer}

A final theme included images or posts that suggested youth and their families reflected on the life skills (i.e., pillars) associated with the virtual sport-based PYD activities. One post was an image of a youth participant and an adult playing indoors. The image also included recognition of one of the pillars of the LAFC program, "integrity." This image suggests youth not only watched the videos but also reflected on the life skill (i.e., pillar) tied to the sport-based PYD activities. Several youth posted photos that mentioned areas of learning centered on transfer whereby they acknowledged they could use the pillars in online school or in sport (see Fig. 6).

\section{Discussion}

Our study had two aims focused on understanding the influence of virtual sport-based PYD programming during the COVID-19 pandemic. First, we sought to explore whether virtual sport-based PYD activities were accessible to a population of socially vulnerable youth and their families during the COVID-19 pandemic. Our findings suggest a large number of youth and families (i.e., $\mathrm{N}=53$ ) engaged with the virtual sport-based PYD activities created by high school youth in the LAFC YLP program. Second, youth and families reported a high level of engagement with an average of three videos over the course of the three-month program. Youth and families indicated the activities were

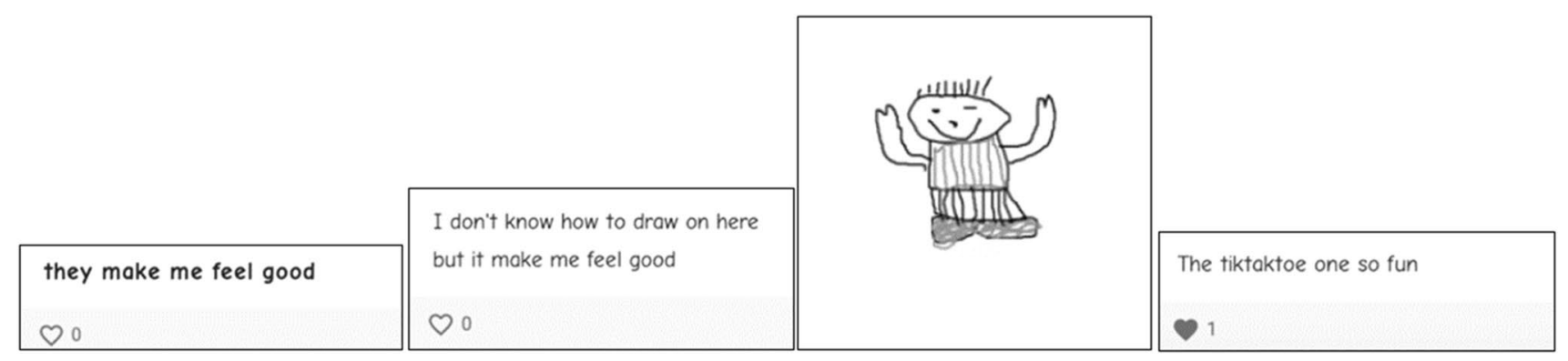

Fig. 2 Positive emotional responses 


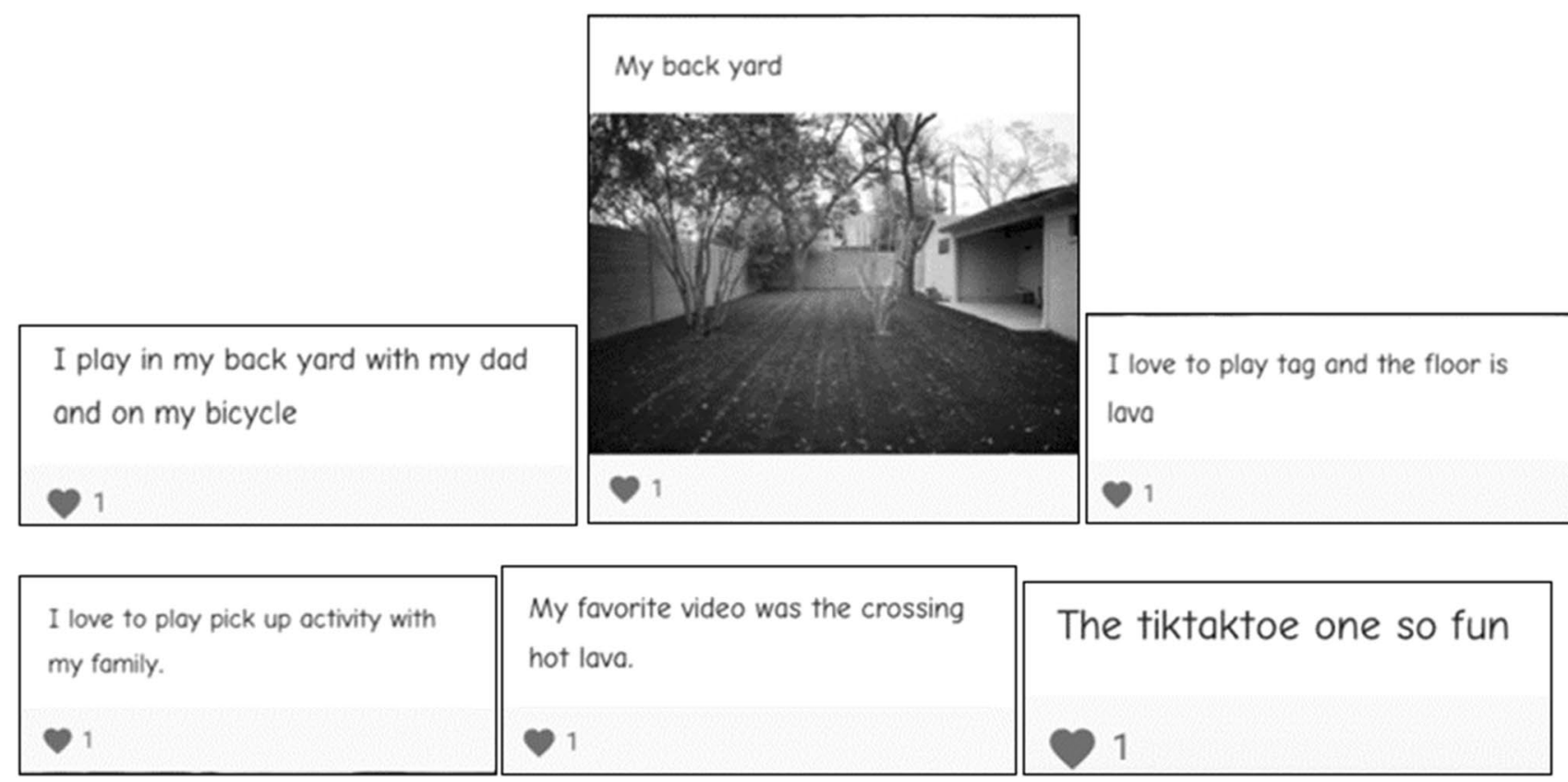

Fig. 3 Positive peer interactions

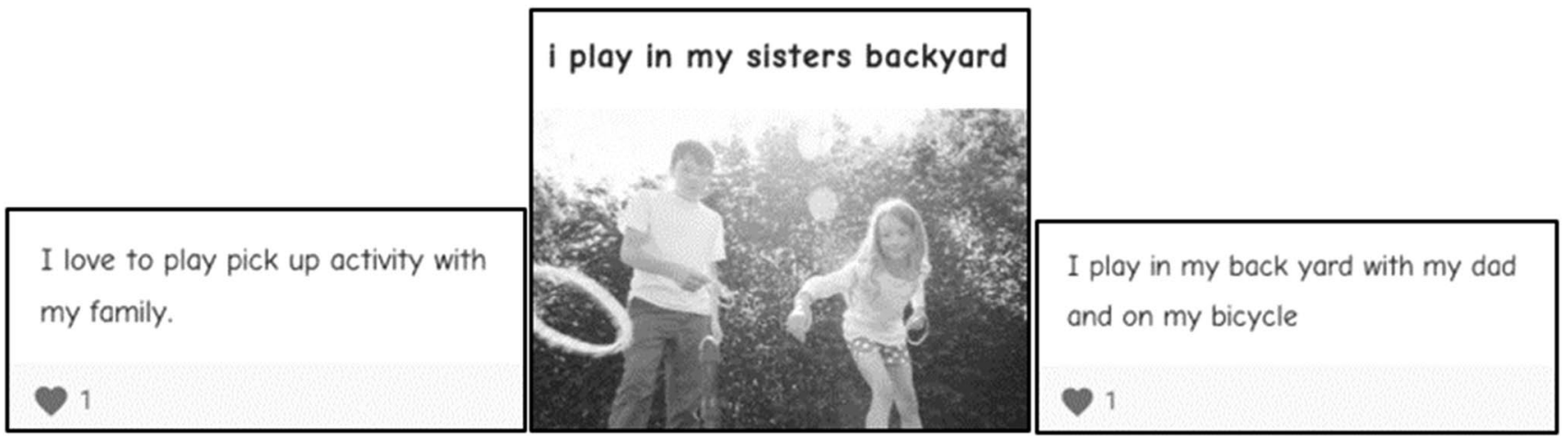

Fig. 4 Engagement with family

enjoyable and challenging. Youth and families also reported value in the activities supporting engagement in physical activity at home, learning skills to utilize in online school settings, and identifying strategies to build relationships in the community.

We also sought to explore the experiences and learning outcomes of youth and families participating in the virtual sport-based PYD activities during the COVID-19 pandemic. Our thematic findings align with ecological systems theory that conceptualizes child development across multiple layers of relationships and contexts (Bronfenbrenner, 1979). For example, Bronfenbrenner (1979) argues that the microsystem is where a child has direct, face-to-face relationships with significant people such as parents, friends, and teachers. Within the microsystem, our results point to the positive influence of sport-based PYD activities in eliciting positive emotional responses, positive peer interactions, and engagement with family members.

In addition, our results suggest the virtual sport-based PYD activities also influenced youth and families within the mesosystem. The mesosystem includes contexts where cross-relationships across multiple settings take place (Bronfenbrenner, 1979). Youth in our study shared experiences of cross-relationships with extended family members and utilization of diverse resources in their environments (i.e., sister's backyard, home environments, and backyards). Bronfenbrenner (1979) also described the macrosystem as the prevailing cultural and economic conditions of the society. Although the virtual sport-based PYD activities did not disrupt or change the economic 

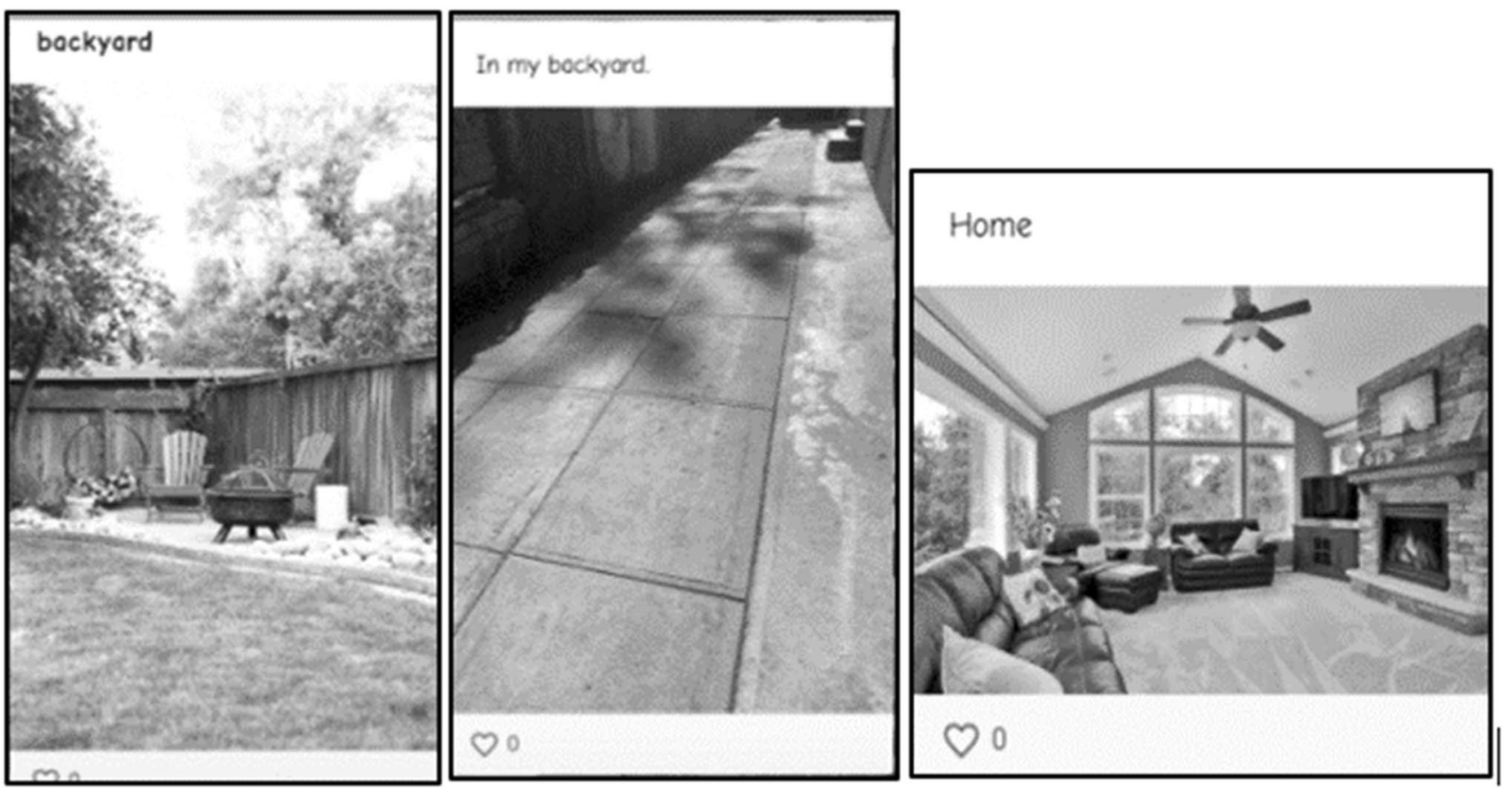

Fig. 5 Utilization of environmental resources

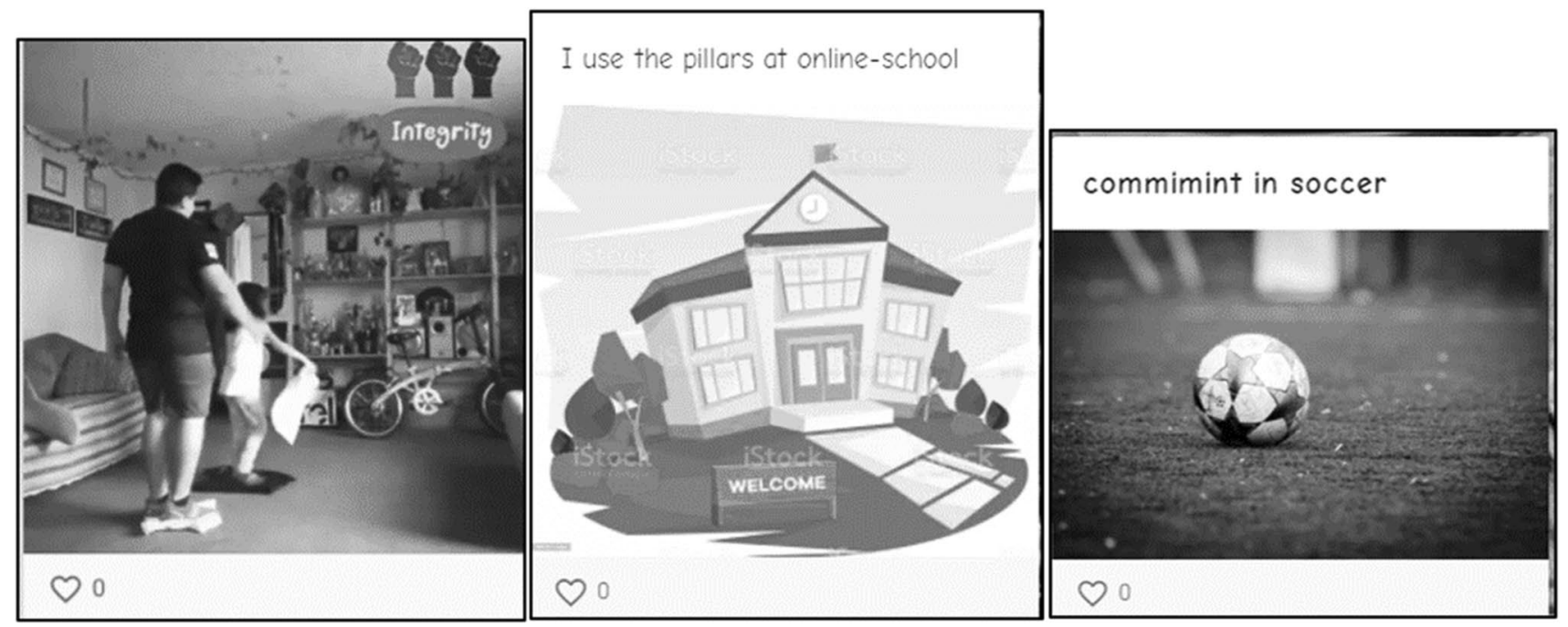

Fig. 6 Life skill transfer

conditions of the youth or families nor COVID-19 regulations, the activities sought to combat a lack of access to sport and play at the structural level (The Aspen Institute, 2020). Our findings indicate partnerships among community-based organizations and sports programs who leverage sport-based PYD for intervention and outreach may have the potential to increase physical activity at the child, family, and community level. Considered together, the virtual sport-based PYD activities during the COVID-19 pandemic positively influenced multiple systems of child development.

We also found pairing the sport-based PYD activities with life skills facilitated participants' reflection on the transfer of life skills to other settings during COVID-19 pandemic. For example in both the online surveys and Padlet, youth and families reported the life skills (i.e., pillars) taught during the activities were transferable to other settings such as online school and the community. Bean et al. (2016) 
have argued that youth not only develop life skills through participation in community sport-based PYD programs, but also learn how to transfer life skills learned in sport to other life domains such as home and school. Our findings advance prior research and contribute to gaps in understanding whether virtual sport programming can support life skill transfer, especially among a socially vulnerable population of youth and families during the COVID-19 pandemic.

We contend that children's potential to develop life skills through virtual sport-based PYD programming is important from a youth development perspective. Prior to the pandemic, scholars estimated that approximately $20 \%$ of youth lack the appropriate social skills necessary for success in school (Blumberg et al., 2008). We found the outreach from the sport-based PYD program, guided by the "Big 3" (Lerner, 2004), may have resulted in what Pierce et al. (2017) define as "life skill transfer." Pierce et al. (2017) developed The Life Skills Transfer Model to frame how sports can facilitate a "process by which an individual further develops or learns and internalizes a personal asset and then experiences personal change through the application of the asset in one or more life domains beyond the context where it was originally learned" (p. 194).

The Life Skills Transfer Model identifies four components that influence life skill transfer. The first component is the individual learner (e.g., internal and external assets). Our findings point to internal and external assets among youth participating in the sport-based PYD activities. For example, youth reported positive emotional and social responses to the activities, and to their external access to environments and spaces that facilitated engagement. The second and third components of the Life Skills Transfer Model include the learning context (e.g., program design features) and transfer contextual factors (i.e., opportunities to learn life skills; Pierce et al., 2017). To date, researchers have found that several sport-based PYD design mechanisms delivered via in-person programming promote positive developmental outcomes for youth (Anderson-Butcher et al., 2013; Pierce et al., 2017; Whitley et al., 2019). However, our results add to what is known about the life skill development that can happen via the delivery of virtual sport-based PYD programming. Our findings suggest virtual programming that includes didactic instruction (i.e., modeling, positive reinforcements, and debriefing) can facilitate meaningful reflections on life skills learned through sport-based PYD.

Finally, Pierce et al.'s (2017) Life Skill Transfer Model suggests individual psychological processes, including the meaningfulness of the learning, influence the transfer of life skills learned through sport. In our study, the community partnership among the Bresee Youth Center and the LAFC professional soccer team, the opportunities to connect with peers during the COVID-19 pandemic, and the cultural pride associated with sport, specifically soccer, may have made the learning activities meaningful for youth and families. In addition, children's opportunity to engage in activities they could do at home during the lockdown may have increased the value of these outreach efforts. These contextual factors are important to evaluate in future research studies to better understand what elements are meaningful in delivering virtual sport-based PYD programming.

\section{Implications}

Findings from our study have several implications for social workers in afterschool and school settings and sport-based PYD leaders. A high level of engagement indicates youth and their families felt these activities were important to engage with and participate in, especially during lockdown. Given youth are at an increased risk for sedentary behaviors during the pandemic (Dunton et al., 2020), virtual sportbased PYD activities may be one intervention strategy practitioners can leverage to reach and engage socially vulnerable youth. Although we did not measure engagement in physical activity, youth and families reported they could do the activities in the home settings. As a result, families and caregivers may have been able to engage their children in sport in play during lockdown, mitigating risks for sedentary behaviors and other psychological and social risks.

Second, findings provide evidence that practitioners can leverage virtual sport outreach to teach social-emotional life skills. Some studies show that children can learn and transfer emotional lessons from TV programs (Wilson, 2008). However, other scholars argue today's youth are saturated with virtual media platforms, games, and outlets, shaping their social, emotional, cognitive, and physical development (Blumberg \& Fisch, 2013). Indeed, researchers argue that poorly designed games and online tools may contribute to a sedentary lifestyle, to increased risks of mental health concerns, or to increase aggressive behaviors (Lieberman et al., 2009; Prot et al., 2012). As a result of the mixed evidence on virtual programs and social-emotional skill development, we advocate for future research focused on distilling what mechanisms within virtual sport-based PYD programming may facilitate positive and sustainable learning and life skill development. We invite researchers to explicitly explore the effects of culturally-responsive virtual programming, such as designing outreach focused on soccer for the Latinx community, when exploring what factors facilitate higher engagement and effective life skill development.

Moreover, our results point to unique opportunities for designing sport-based PYD programming and the development of family-community-sport partnerships. Our study encourages partnerships among families, community-based centers, and professional sport teams to co-construct community outreach efforts. We also see value in designing PYD programs that utilize a ladder of leadership approach 
whereby older youth in the community, who look like and come from a similar background as the younger youth in their community, design and deliver the sport-based PYD activities in culturally-responsive ways (Bates et al., 2020). Older youth may benefit by modeling and practicing the skills; suggesting sport-based PYD programming reaches multiple populations. High school youth also have opportunities to be positive role models and give back to their communities. A final implication of this work is the accessibility of the activities to youth and families with fewer resources, especially during the COVID-19 pandemic. Social workers and sport leaders can work to design sport-based PYD activities that do not require resources, equipment, or outdoor spaces. We contend virtual sport-based PYD may have the potential to support engagement in physical activity and relationship building at home.

Notably, our study also has several limitations. First, we did not capture demographic indicators of the youth and families that participated in the activities. We are therefore unable to describe the specific characteristics of youth who may have benefited from the virtual sport-based PYD activities. Second, we may have seen a high-level social desirability bias in the responses from youth and their families. Youth and their families who enjoyed the activities may have been those more likely to complete the surveys or to report a positive experience to obtain the associated prizes or incentives. Third, the use of Padlet and language barriers may have inhibited some youth and families from participating in the study. On the other hand, the use of Padlet offered a unique way to collect data, allowed youth to more freely express themselves, and contributed to our ability to elevate the voices, experiences, and perceived learning of the youth participants.

\section{Conclusion}

This study sought to explore whether virtual sport-based PYD activities were accessible to a population of socially vulnerable youth and their families during the COVID-19 pandemic. We also examined the lived experiences of youth during the pandemic and their learning outcomes. Our findings indicated virtual sport-based PYD activities were accessible, enjoyable, and challenging for the youth participants. We also found virtual sport-based PYD activities facilitated positive emotional responses, positive peer interaction, engagement with family, and utilization of environmental resources during the COVID-19 pandemic. Importantly, our findings suggest virtual sport-based PYD activities facilitate life skill transfer; an important developmental mechanism for learning in lieu of the decreased opportunities for sport and social interaction during the COVID-19 pandemic. Our findings present future opportunities for social workers to aid in the delivery of virtual social-emotional learning programs and development of unique school-community-sport partnerships.

Funding There was no grant funding attached to this work.

\section{Declarations}

Conflict of interest We have no conflicts of interest to disclose.

Ethical Approval All procedures performed in studies involving human participants were in accordance with the ethical standards of the institutional and/or national research committee and with the 1964 Helsinki Declaration and its later amendments or comparable ethical standards. The study was approved by the Institutional Review Board at Texas Christian University.

Informed Consent Informed consent was obtained from legal guardians.

\section{References}

Center for Disease Control. (2021, May 13). Adolescent and school health. Retrieved from https://www.cdc.gov/healthyyouth/data/ yrbs/

Anderson-Butcher, D. (2019). Youth sport as a vehicle for social development. Kinesiology Review, 8(3), 180-187

Anderson-Butcher, D., Riley, A., Amorose, A., Iachini, A., \& WadeMdivanian, R. (2013). Maximizing youth experiences in community sport settings: The design and impact of the LiFE Sports Camp. Journal of Sport Management, 28(2), 236-249

Bachman, R. (2020, April 7). Coronavirus could cause youth sports recession. https://www.wsj.com/articles/coronavirus-could-causeyouth-sports-recession 11586260815

Bates, S., Anderson-Butcher, D., Ferrari, T., \& Clary, C. (2020). A comparative examination of how program design components influence youth leadership-skill development. Journal of Youth Development, 15(6), 91-115

Bean, C., Kendellen, K., \& Forneris, T. (2016). Moving beyond the gym: Exploring life skill transfer within a female physical activitybased life skills program. Journal of Applied Sport Psychology, 28(3), 274-290

Blumberg, F. C., \& Fisch, S. M. (2013). Introduction: Digital games as a context for cognitive development, learning, and developmental research. New Directions for Child and Adolescent Development, 2013(139), 1-9

Blumberg, S. J., Carle, A. C., O’Connor, K. S., Moore, K. A., \& Lippman, L. H. (2008). Social competence: Development of an indicator for children and adolescents. Child Indicators Research, 1(2), 176

Botvin, G. J., \& Griffin, K. W. (2004). Life skills training: Empirical findings and future directions. Journal of Primary Prevention, 25(2), 211-232

Bouchard, C., Blair, S. N., \& Haskell, W. L. (2012). Physical activity and health. Human Kinetics.

Bresee Youth Center. (2020). About us. Retrieved from https://www. bresee.org/who-we-are/

Bronfenbrenner, U. (1979). The ecology of human development: Experiments by nature and design. Harvard University Press. 
Camiré, M., Trudel, P., \& Forneris, T. (2012). Coaching and transferring life skills: Philosophies and strategies used by model high school coaches. The Sport Psychologist, 26(2), 243-260

Dunton, G. F., Do, B., \& Wang, S. D. (2020). Early effects of the COVID-19 pandemic on physical activity and sedentary behavior in children living in the US. BMC Public Health, 20(1), 1-13

Durlak, J. A., Weissberg, R. P., \& Pachan, M. (2010). A meta-analysis of after-school programs that seek to promote personal and social skills in children and adolescents. American Journal of Cоттиnity Psychology, 45(3), 294-309

Hermens, N., Super, S., Verkooijen, K. T., \& Koelen, M. A. (2017). A systematic review of life skill development through sports programs serving socially vulnerable youth. Research Quarterly for Exercise and Sport, 88(4), 408-424

Holt, N. L., Neely, K. C., Slater, L. G., Camiré, M., Côté, J., FraserThomas, J., ... Tamminen, K. A. (2017). A grounded theory of positive youth development through sport based on results from a qualitative meta-study. International Review of Sport and Exercise Psychology, 10(1), 1-49

Janssen, I., \& LeBlanc, A. G. (2010). Systematic review of the health benefits of physical activity and fitness in school-aged children and youth. International Journal of Behavioral Nutrition and Physical Activity, 7(1), 1-16

Jones, G. J., Edwards, M. B., Bocarro, J. N., Bunds, K. S., \& Smith, J. W. (2017). An integrative review of sport-based youth development literature. Sport in Society, 20(1), 161-179

Kelly, A. L., Erickson, K., \& Turnnidge, J. (2020). Youth sport in the time of COVID-19: Considerations for researchers and practitioners. Managing Sport and Leisure. https://doi.org/10.1080/23750 472.2020.1788975

Lerner, R. M. (2004). Liberty: Thriving and civic engagement among American youth. Sage.

Lieberman, D. A., Fisk, M. C., \& Biely, E. (2009). Digital games for young children ages three to six: From research to design. Computers in the Schools, 26(4), 299-313

Lincoln, Y. S., \& Guba, E. G. (1986). But is it rigorous? Trustworthiness and authenticity in naturalistic evaluation. New Directions for Program Evaluation, 1986(30), 73-84

Margaritis, I., Houdart, S., El Ouadrhiri, Y., Bigard, X., Vuillemin, A., \& Duché, P. (2020). How to deal with COVID-19 epidemicrelated lockdown physical inactivity and sedentary increase in youth? Adaptation of Anses' benchmarks. Archives of Public Health, 78, 1-6

McMahon, S. D., Todd, N. R., Martinez, A., Coker, C., Sheu, C. F., Washburn, J., \& Shah, S. (2013). Aggressive and prosocial behavior: Community violence, cognitive, and behavioral predictors among urban African American youth. American Journal of Community Psychology, 51(3-4), 407-421
Nadel, J. H. (2014). Fútbol! Why soccer matters in Latin America. University Press of Florida

Pierce, S., Gould, D., \& Camiré, M. (2017). Definition and model of life skills transfer. International Review of Sport and Exercise Psychology, 10(1), 186-211

Pierce, S., Kendellen, K., Camiré, M., \& Gould, D. (2018). Strategies for coaching for life skills transfer. Journal of Sport Psychology in Action, 9(1), 11-20

Prot, S., McDonald, K. A., Anderson, C. A., \& Gentile, D. A. (2012). Video games: Good, bad, or other? Pediatric Clinics, 59(3), 647-658

Rundle, A. G., Park, Y., Herbstman, J. B., Kinsey, E. W., \& Wang, Y. C. (2020). COVID-19-related school closings and risk of weight gain among children. Obesity, 28(6), 1008-1009

Sanderson, J., \& Brown, K. (2020). COVID-19 and youth sports: Psychological, developmental, and economic Impacts. International Journal of Sport Communication, 13(3), 313-323

Sherwin, I. (2020, May 13). Coaching during the pandemic presents a unique challenge. https://pess.blog/2020/05/18/coaching-during-the-pandemic-presents-a-unique-challenge-dr-ian-sherwin/

Spruit, A., Assink, M., van Vugt, E., van der Put, C., \& Stams, G. J. (2016). The effects of physical activity interventions on psychosocial outcomes in adolescents: A meta-analytic review. Clinical Psychology Review, 45, 56-71

The Aspen Institute. (2019, March 10). Youth sports facts: Sports participation and physical activity rates. Retrieved from https://www. aspenprojectplay.org/youth-sports-facts/participation-rates

The Aspen Institute. (2020, January 19). Project play state of play 2020: Pandemic trends. Retrieved from https://www.aspenproje ctplay.org/state-of-play-2020/pandemic-trends

Whitaker, A. A., Baker, G., Matthews, L. J., McCombs, J. S., \& Barrett, M. (2019, May 12). Who plays, who pays? Funding for and access to youth sports. https://www.rand.org/pubs/research_repor ts/RR2581.html

Whitley, M. A., Massey, W. V., Camiré, M., Boutet, M., \& Borbee, A. (2019). Sport-based youth development interventions in the United States: A systematic review. BMC Public Health, 19(1), $1-20$

Wilson, B. J. (2008). Media and children's aggression, fear, and altruism. The Future of Children, 18(1), 87-118.

Publisher's Note Springer Nature remains neutral with regard to jurisdictional claims in published maps and institutional affiliations. 